http://artnodes.uoc.edu

\title{
La gestión matemática de la sinceridad Algoritmos y veridicción
}

\author{
Jorge Luis Marzo Pérez \\ BAU Centre Universitari de Disseny de Barcelona
}

Fecha de publicación: julio de 2019

\section{Cita recomendada}

Marzo Pérez, Jorge Luis. 2019. «La gestión matemática de la sinceridad. Algoritmos y veridicción». En «Tras la posverdad». Artnodes, n. ${ }^{\circ}$ 24: 1-12. UOC. [Fecha de consulta: dd/ mm/aa] http://dx.doi.org/10.7238/a.v0i24.3306

\footnotetext{
Cos textos publicados en esta revista están sujetos -si no se indica lo contrario- a una licencia de
Reconocimiento 4.0 Internacional de CreativeCommons. La licencia completa se puede consultar en https://creativecommons.org/licenses/by/4.0/deed.es_ES.
}

\begin{abstract}
«Después de la instrucción, en cambio, unas compañías charlaban animadamente con otras, gritando por las ventanas abiertas de los acantonamientos frases sobre el concepto de la verdad coherente, juicios analíticos y sintéticos a priori y razonamientos sobre la existencia in se; éste era ya el nivel alcanzado por la inteligencia colectiva, cuyo trabajo mental condujo a elaborar leyes de filosofía, hasta que un batallón llegó a un solipsismo total, proclamando que fuera de él no existía concretamente nada» (Lem 2005, 18).
\end{abstract}

\section{Los hechos}

En octubre de 1995, el Dr. Joseph Bonuso, investigador vinculado a la New York University Law School, envió un comunicado de prensa a funcionarios, jueces y decanos de escuelas de derecho, anunciando que él, junto a 150 informáticos y abogados, había desarrollado una solución a la crisis de confianza en la jurisprudencia estadounidense. Lo llamó The Solomon Project. Solomon era el nombre de un programa de Inteligencia Artificial (IA) capaz de «deliberar» sobre los hechos y las pruebas de un caso a fin de emitir un veredicto «incontaminado», eliminando así todo posible error de jurados y jueces. En paralelo a la introducción en el programa de toda la literatura jurisprudente americana, el sistema procesaba los análisis de estrés de voz y telemetría de polígrafo de todos los testigos y togados, con el objetivo de establecer su sinceridad, descartando así la desigualdad del aparato judicial en relación con la raza, el sexo, la religión o la posición financiera de los acusados.

El comunicado daba fe de las pruebas concluyentes realizadas por el nuevo algoritmo a partir de los materiales documentales procedentes de una serie de juicios que habían despertado notable atención y controversia pública pocos años antes, como los de Mike Tyson, William Kennedy-Smith, Klaus Von Bulow y Rodney King. Asimismo, se anunciaba que el programa estaba trabajando en el caso de 0.J. Simpson, el famoso deportista que acababa de ser absuelto por el asesinato de su ex esposa y de la amante de esta. Al hacerse público el resultado del algoritmo, que declaraba a Simpson 


\section{artnodes}

http://artnodes.uoc.edu

La gestión matemática de la sinceridad...

culpable, se despertó un gran interés en entornos jurídicos y medios de comunicación. La cadena CNN, por ejemplo, grabó un extenso reportaje en el laboratorio informático donde Solomon procesaba los datos. En él, se observa a un grupo de expertos trabajando frente a sus ordenadores, mientras Bonuso responde a las preguntas de los periodistas.

Al día siguiente de la emisión, el artista Joey Skaggs hacía pública una nota en la que manifestaba que todo había sido un fake, un hoax, una «veroficción» (Marzo 2018), una broma creativa orquestada por él junto a algunos amigos y colegas. No había ningún algoritmo milagroso, ni vinculación alguna con la universidad; las oficinas del proyecto se habían montado días atrás con ordenadores corrientes, en cuyas pantallas parecían correr sofisticados programas; los operarios eran estudiantes haciendo de actores. En la nota del exposé (todo fake, a diferencia del mero mentir, debe revelarse en algún momento), Skaggs señalaba: «Es indignante que los casos civiles y penales se desarrollen ante nuestros ojos como si fueran partidos deportivos. La verdad ya no proporciona la llave a la justicia», y se preguntaba sobre qué sistema había que adoptar para conseguir una justicia real y asequible para todos (Skaggs 1996). La CNN y otros medios se vieron obligados a «disculparse» públicamente. El debate social posterior fue vivo, en parte sobre la base de que las retractaciones de los medios despertaban dos cuestiones verdaderamente competentes: el haber creído que era posible una justicia digital, o el haber creído que era buena. Y todo ello iluminado por una impostura, cuyos efectos fueron advertir por un momento del complejo tejido que forman binomios como verdadero y falso, formato y verosimilitud, veracidad y sinceridad. La tradición de lo falsario se basa en el diseño de métodos procaces y eficaces de exposición de la trama en la que se conducen los juegos de la veridicción, del «decir veraz». Si ello lo hacemos extensivo al universo judicial, los efectos de la exposición son, seguro, más reveladores. Las siguientes páginas pretenden hacer un recorrido paralelo al de Skaggs sobre los factores que arman hoy la relación entre verdad, justicia e inteligencia artificial y que pueden condicionar, si no lo están haciendo ya, nuestros modos de confiar y sospechar. La diferencia con el bulo orquestado por el artista americano es que lo que en él era distopía hoy es una realidad en pleno desarrollo.

\section{El contexto}

Efectivamente, existe una extensa percepción de que el principal lugar en el que se imparte la justicia, los tribunales, están excesivamente hipotecados por una serie de técnicas y formatos procesales que favorecen una constante manipulación argumental, que impide a menudo dirimir con propiedad la verdad de los hechos encausados. En realidad, se trata de una vieja percepción que podemos rastrear hasta las primeras formulaciones jurídicas del mundo clásico grecolatino: ¿cómo se establece la sinceridad de un testigo, ya no digamos del acusado?, ¿cómo se fija la imparcialidad de un juez 0 de un jurado?

Emplazar el tema de la verdad en un tribunal supone afrontar una reflexión con evidentes consecuencias de competencia pública y política. No solamente es un tribunal la sede final y última de la economía de la verdad y de su régimen, sino que sobre todo es allí en donde los efectos de este principio implican la suerte o desgracia pública flagrante de la vida social de cualquiera. Es esta condición de competencia pública radical la que hace de todo proceso jurídico el principal foco de atención a la hora de analizar las transformaciones intensas y profundas de la condición de verdad en nuestra vida común actual, un proceso que ha venido a definirse en términos de «régimen de la posverdad».

En el Congreso Internacional Interface Politics (After Post-Truth) ${ }^{1}$ se abogó desde un principio por enfocar el fenómeno de la posverdad mediante un criterio de competencia pública que se sustrajese en lo posible al debate del efecto Roshomon, en recuerdo al título de la película de Akira Kurosawa en la cual diferentes sujetos describen el mismo hecho de forma distinta sin que por ello ninguna versión sea falsa; debate eterno y no del todo útil de la verdad, sostenido únicamente sobre la «constatación» de la verdad subjetiva como factor depresor de una construcción colectiva de lo cierto. El Congreso vino a promover nuevos ingredientes: ¿Cómo se puede afrontar el intenso y ruidoso debate sobre la transformación acelerada de los mecanismos de autoridad y consenso en la construcción y legitimación de lo cierto, de lo veraz, cuando es precisamente la percepción cacofónica de las voces (de kakós, feo, engorroso) la que da pie a la búsqueda acelerada de sistemas «objetivos» que permitan suprimir todo ruido subjetivista, que consigan -como Skaggs iluminaba mediante su parodia- «fijar» la verdad más allá de toda duda?

Cualquier análisis o discusión del papel de las interfaces en relación con su agencia o competencia pública no puede dejar de atender una cuestión como el del régimen de verdad, operado hoy

\footnotetext{
1. El presente número 24 de Artnodes tiene su origen en el II Congreso Internacional Interface Politics (After Post-Truth), celebrado el 28,29 y 30 de noviembre de 2018 en Barcelona. Chairs: J.L. Marzo y B. Brusadin. Organizado por GREDITS (Grup de Recerca en Disseny i Transformació Social) y BAU Centre Universitari de Disseny, con la colaboración de HANGAR Centre de Producció i Recerca d'Arts Visuals y MACBA, Museu d'Art Contemporani de Barcelona. Web del congreso: http://www.gredits.org/interfacepolitics/es/. Actas de la primera edición: http://www.gredits.org/wp-content/uploads/2016/11/Publicacions_Gredits_04_V2_web.pdf
} 


\section{artnodes}

http://artnodes.uoc.edu

La gestión matemática de la sinceridad...

por máquinas. ${ }^{2}$ Las interfaces, en la medida en que se vuelven cada vez transparentes, ubicuas, estandarizadas, cómodas e invisibles, se vuelven inteligentemente «naturales» en la percepción de los operarios e impide un debate competente sobre la condición de su naturalidad, sobre la transformación de los lenguajes colectivos, o acerca de la reconfiguración del sujeto productivo. Los artículos de Distelmeyer, de Manuel y Shanbaum publicados aquí analizan con gran propiedad los efectos de la condición inasible de las interfaces, bien en clave de depresentación (Distelmeyer), en referencia a las consecuencias patólógicas de la condición ubicua o panóptica de la interfaz (de Manuel), o en relación con las distopías planteadas por ciertas ramas del Media Art (Shanbaum). Recientes análisis ya describen las relaciones entre la nueva economía y la ecología neuronal de la atención, con un papel de las interfaces mucho más relevante de lo esperado, precisamente por la naturalización y adopción de la lógica computacional en la vida social (Berardi 2017; Citton 2014).

La naturalización de la presencia, causas y efectos de la interfaz ha estado presente en todos los debates sobre el sistema público de credibilidad. Una de las principales cuestiones involucradas en la constitución y transformación de los regímenes de verdad son los aparatos de veridicción, los dispositivos autorizados para establecer la sinceridad y la veracidad. En breve abordaremos el tema. Otros asuntos, como la configuración de la objetividad en la imagen y en el lenguaje técnico son también relevantes para comprender las «mutaciones» en la forma que tenemos de fijar lo objetivo. Pold analiza en su texto la «pulsión realista» de todo interfaz, especialmente cuando este está integrado como metalenguaje, así como las reacciones que esa condición despierta en determinados círculos artísticos y activistas. Jordana y Rispoli se ocupan también de este aspecto central de la genealogía de la veridicción: cómo las interfaces «manifiestan» la verdad a partir de una particular organización de lo sensible; cómo los datos, licuados en el amable tejido de las interfaces, acaban siendo asumidos como vehículos de una mayor verdad que los cuerpos de los que han sido extraídos. En análoga dirección escribe León-Mendoza, en el sentido de cómo la imagen-dato de la videovigilancia adquiere una naturaleza veridictiva, -una «plusvalía semiótica»- por encima de lo real, que afecta tanto al sistema judicial como a la forma de «predecir el pasado» -en una línea, cabría añadir, no alejada de la explorada en su día por Carlo Ginzburg, respecto al impacto de la investigación científica de patrones en la forma de comprender la factualidad (Ginzburg 1980).

El desencarnamiento o desahucio de la imagen actual, y los efectos que ello tiene sobre su carácter fáctico, no sólo debe vincularse a las arraigadas y perdurables tesis platónicas sobre el phantasma, sino que hoy se propone como una nueva plataforma sobre la que dirimir el conflicto entre presentación y representación, entre fidelidad y fetichización. Esa aproximación es propuesta por González-García bajo ciertas perspectivas procedentes del arte contemporáneo, siempre regidas por la constante tensión propositiva entre verdad y ficción. García, por su parte, plantea cómo ciertas ópticas artísticas resisten las invasiones algorítmicas de la privacidad y de control destinadas a establecer qué «grado de libertad» es el más adecuado.

El debate sobre la «adecuación» se revela capital para comprender los cambiantes procesos sobre la objetividad. Si hacemos caso a Nietzsche cuando dice que la verdad es un simple formato, hoy es necesario atender a los modos en que las «opciones» se manifiestan, precisamente mediante interfaces de carácter utilitarista y subjetivista, en parte deudoras de la pragmática lingüística. Tanto Van de Keulenaar como de Valk, en sus sendos artículos, exploran desde ópticas distintas la configuración de esas opciones. La primera lo hace a través de la exploración de las pugnas existentes entre las plataformas enciclopédicas, digitales a fin de establecer el grado de verdad de los datos publicados; la segunda, mediante el análisis de un videojuego de 1985 que proponía una experiencia para combatir la desinformación y recuperar una agencia social interrogativa sobre el papel de los medios de comunicación, actores cada vez mayores en la nueva economía de la credibilidad, tal y como demuestra el artículo de Cosentino y Alikasifoglu respecto a la tragedia siria.

La intención de este número de Artnodes es ofrecer algunas exploraciones fenomenológicas de un complejo proceso que tiene que ver con las mutaciones, tanto de los mecanismos o formatos de percepción de la realidad, como de los modos que los discursos tienen de presentarse en términos de competencia pública. Y efectivamente, el concepto de veridicción tiene no solamente un papel central en estas cuitas, sino que permite articular líneas argumentales que cuestionen lo que Joey Skaggs venía a denunciar en su parodia: lo que queda ausente en la aplicación del lenguaje matemático sobre la veridicción es la misma idea de cuerpo social, y de rebote, la posibilidad de pensar la falibilidad de nuestros procesos hermenéuticos, «porque de ellos depende la sabiduría de una comunidad» (Eco 1998, 18-19). ¿Es un sistema lógico -como los algoritmos inteligentes- una forma de comunidad? ¿Cuáles serían sus miembros y a qué valores comunes estarían sujetos? Ahí hay apelaciones de los padres de la escuela pragmatista respecto a la competencia pública -John Dewey o C.S. Pierce, de gran influencia en los debates lingüísticos contemporáneos, que abogan por la necesaria productividad de los discursos-, cuando plantearon la «community of inquiry» (Dewey 2008) 0 la utilidad de que toda opinión deba influir y ser influenciada en el marco de comunidades, no simplemente entre meros individuos (Pierce 1877).

\footnotetext{
2. Interface Politics es un congreso organizado por BAU desde 2016 con el objetivo de analizar y discutir el papel de las interfaces, como mecanismo comunicacional y lingüístico, y como dispositivo con agencia política, mercantil y laboral. Una interfaz se define hoy a grandes rasgos como un sistema de comunicación que sirve para traducir realidades físicas en lenguajes técnicos y viceversa, o para hacer compatibles lenguajes técnicos distintos (Marzo 2015).
} 


\section{artnodes}

http://artnodes.uoc.edu

La gestión matemática de la sinceridad...

Lo que en Skaggs era burla en 1995, mofándose de las pulsiones tecnoutilitaristas de la comunidad de justicia, veinte años más tarde se convertía en animada noticia judicial. En 2016 se hizo público un estudio académico realizado entre diversas universidades británicas y estadounidenses que analizaba el impacto de la IA (en concreto, de las recientes técnicas de natural language processing y machine learning) en las decisiones judiciales (Aletras et al. 2016). El trabajo afirmaba la idoneidad del uso de patrones extraídos mediante algoritmos de aprendizaje computacional para predecir el resultado de un juicio, después de ser aplicados a los textos procedentes de los casos procesados por el Tribunal Europeo de Derechos Humanos. El resultado del trabajo fue un acierto en el fallo del $79 \%$, e invitaba a la comunidad jurídica a tener presente este tipo de sistemas a la hora de utilizar futuros mecanismos de investigación legal.

El estudio presentaba también las dificultades en la aplicación de la IA, dadas las condiciones y efectos de la interacción humana y las complejidades morales resultantes. En el mismo sentido se manifestaba la Carta Ética aprobada por la Comisión Europea para la Eficiencia de la Justicia de 2018 sobre la aplicación de estos sistemas (CEPEJ 2018). Numerosos organismos y autoridades judiciales, no obstante, ya están adoptando estos algoritmos a la vista de la reducción de costes laborales en una administración de Justicia con poco presupuesto. En España, el Consejo General del Poder Judicial y el Consejo General de la Abogacía anunciaron en 2017 y 2018, respectivamente, la paulatina inserción de la IA en las fases documentales de trabajo de abogados, jueces y procuradores (CGPJ 2017; CGA 2018). El Ministerio de Justicia estonio está financiando la creación de un sistema de IA que pueda dirimir disputas legales de menos de $7000 €$ (Niler 2019), y en Estados Unidos, empresas como Equivant ofrecen servicios de procesamiento «inteligente» de datos, destinados tanto a la gestión de causas legales como a la valoración de riesgos en la toma de decisiones de los jueces relativas a la prisión preventiva, la libertad bajo fianza o la libertad condicional (Tashea 2017). ${ }^{3}$

¿Estamos, por tanto, frente a una potente actualización de los dispositivos de veridicción heredados del cientifismo positivista en forma de sistemas autorreferentes, 0, por el contrario, acaso encaramos una mutación mayor?

\section{La veridicción o el «decir veraz»}

La veridicción es el dispositivo sociolingüístico que establece el régimen de sospechas y confianzas en determinados dominios; es una suerte de contrato que determina en qué condiciones decimos la verdad o aceptamos como verdaderos los discursos ajenos. Para Michel Foucault, quien formularía el asunto con solvencia a partir de su lección inaugural en el Collège de France en 1970, pero sobre todo desde su libro El orden del discurso, la sinceridad (el decir veraz) es un balancín entre la construcción social de una interioridad (el sujeto) y su recepción exterior; entre la convención que se requiere para generar en los sujetos la íntima convicción de estar expresando la verdad y la autorización social de que, en efecto, el sujeto dice la verdad. La veridicción no dictamina lo que es o no verdad factual, su radio de acción cubre más bien la configuración social de su relato como esquema de competencia pública: cómo el lenguaje sirve para armar ciertos modos jurisprudentes y de producción de sentido que acaban configurándose como regímenes de verdad. Umberto Eco insistiría mucho en ello: una cosa es el «principio de verdad» y otro el de «confianza», aunque dependan el uno del otro (Eco 1996, 98-102). Para Foucault, la veridicción traza la arquitectura de la sinceridad, no de la veracidad. La veridicción se constituye mediante «los mecanismos y las instancias que permiten distinguir los enunciados verdaderos o falsos, la manera de sancionar unos y otros; las técnicas y los procedimientos que son valorizados para la obtención de la verdad; y el estatuto de aquellos encargados de decir qué es lo que funciona como verdadero» (Foucault 1980, 187). La veridicción, por consiguiente, se ocupa de cómo los formatos con los que la veracidad se presenta acaban creando la verosimilitud y veracidad necesarias para constituirse jurídicamente, teniendo siempre presente que la veridicción es la propia historia de la verdad y de su persuasión, sujeta permanentemente a los tiempos históricos. También Greimas y Courtés abundaron en su día sobre la necesidad de pensar la veridicción en términos de dispositivos persuasivos: «La producción de la verdad corresponde al ejercicio de un hacer cognoscitivo particular, el hacer parecer verdad, que puede ser denominado, sin ningún matiz peyorativo, hacer persuasivo» (Greimas y Courtés 1982, 433).

Foucault parte del estudio de las «formas de la sinceridad», presentes tanto en la Grecia clásica como en la formación y despliegue del discurso judeocristiano hasta la Edad Media. Marcel Detienne ya exploró en 1967 cómo el enunciado griego de la verdad fue variando a lo largo del tiempo. En sus inicios tenía mucho que ver con la búsqueda de la «palabra eficaz», vinculando a las musas en esa construcción. Así, la «palabra eficaz», la alétheia, fundamentalmente en boca del poeta, «no era la concordancia de la proposición con su objeto, tampoco la concordancia de un juicio con otros juicios» sino un «don de videncia» del pasado y del futuro, en el que se conjugaban los modos de justicia autorizados por el poder y los procederes adivinatorios. Sin embargo, más adelante, por ejemplo, en las formulaciones realizadas a partir de Simónides, el poeta deja de poseer una mera condición religiosa y adivinatoria para dejar paso a la doxa, a lo que llamaríamos «opinión común», que se mantendría competente gracias a la aparición de la mnemotecnia, de la técnica de patrones

3. Véase: https://www.equivant.com/. 


\section{artnodes}

http://artnodes.uoc.edu

La gestión matemática de la sinceridad...

literarios y visuales que hacen posible la conservación de la memoria y que permiten la vertebración de unas genealogías, cuyos patrones ayuden a interpretar los hechos del presente (Detienne 2004, 65-103).

No obstante, la diferencia sustancial en el análisis de Foucault es su intención de cartografiar las partes obsolescentes de ese formato una vez llegada la modernidad, en especial en lo relativo a la deconstrucción de la interioridad clásica. Para ello, Foucault traza la genealogía de determinadas formas de enunciación vinculadas a la construcción de la interioridad, que podríamos dividir en dos: una, que articula la idea de una conciencia íntima (ejemplificada en el «examen de conciencia»), y otra que se andamia a través de la expresión pública de la misma, como es, por ejemplo, la «parresía», el «hablar con franqueza», ese «decir eficaz» propuesto por la retórica clásica (Revel 2018). Foucault define la parresía como aquella «actividad verbal en la cual un hablante expresa su relación personal a la verdad, y corre peligro porque reconoce que decir la verdad es un deber para mejorar 0 ayudar a otras personas (tanto como a sí mismo)». En parresía, «el hablante usa su libertad y elige la franqueza en vez de la persuasión» (Foucault 2001, 19-20). Esto es, la subjetividad y la intimidad se configuran como espacios aparentemente predispuestos para el surgimiento de la sinceridad, aún a pesar de que la exterioridad pueda violentarlos en grado sumo, al poner en cuestión el orden colectivo y proponer nuevos marcos interpretativos. Al mismo tiempo, en la sinceridad encontramos las formas para construirnos como sujetos de verdad.

El reconocimiento social de la conciencia íntima del sujeto es central en este asunto. Foucault da cuenta de su modulación social: por ejemplo, el discurso del loco en la Edad Media es considerado nulo y sin valor, al no disponer de una conciencia «competente», pero, al mismo tiempo, su palabra es enmarcada a menudo en la esfera de la predicción mágica, al enunciar verdades ocultas o predecir fenómenos gracias a su ingenuidad, no contaminada por la doxa social. Una condición similar asumirían los niños, los ancianos o los animales, como podemos aún constatar en las figuras empleadas por los ventrílocuos, ya que se sugieren exentos de la moralidad y del lenguaje social imperantes, y se muestran por consiguiente más dispuestos a «cantar las verdades» sin el recato de las sanciones morales, a la vez que reciben, por el contrario, el aplauso y reconocimiento del público. Este aspecto predictivo de la verdad no es exclusivo de una condición desgajada de la conciencia (de lo que nos ocuparemos en breve), ya que Foucault también lo emplaza en ciertos poetas griegos, cuyos discursos «verdaderos» se habían ganado la potestad (como en el caso de los fantasmas) de generar «respeto y terror» mediante rituales enunciativos bien establecidos que anunciaban lo que estaba por venir y que contribuían así a su realización, arrastrando consigo la adhesión de los hombres hacia su propio destino (Foucault 2004).

Si, por un lado, la interioridad plasmada por la conciencia íntima del decir veraz deviene parte esencial del contrato de veridicción, por otro lado, aparece la articulación de unos mecanismos y formatos exteriores capaces de establecer la verosimilitud de la franqueza. Foucault se ocupa de estos mediante el estudio de varios dispositivos, como el sacramento católico de la confesión o la confesión judicial. Procedimientos como la tortura acabaron configurándose como formatos necesarios para incitar al interrogado, a ese sujeto que necesita ser preguntado para revelar la verdad y lograr así la absolución. Para Foucault, la confesión establece con claridad la voluntad del poder en distinguir la «aserción» (verdadera o falsa) del acto de decir o escuchar la verdad, la «veridicción» (Foucault 2014, 29); esto es, establece equivalencias entre la interioridad y la exterioridad, entre la sinceridad y la veracidad. Por su parte, el recurso al testimonio (la adtestatio rei visae) dado por un testigo ocular, reflejaba la capacidad otorgada a alguien -de quien no se duda de su interioridad o sinceridad (pues no se juega el cuello) - de proporcionar veracidad frente a la fábula engañosa, abriendo las puertas a la aparición del pathos, de la emoción, y por lo tanto, de una fábula mucho mayor, cuyo epítome sería la demostración científica o forense construida como relato.

La modernidad científica o paracientífica actualizará los mecanismos para la extracción de la sinceridad: la máquina de la verdad (polígrafo), la hipnosis, el pentotal o suero de la verdad, o el propio psicoanálisis se manifestaron en su día como aparentes inductores de un relato más o menos fiable de la conciencia más secreta y sincera, tanto que los propios sujetos la desconocen y que es necesario hacer aflorar. Este aspecto es importante, pues los mecanismos exhibidores de esta conciencia secreta solo pueden legitimarse en la medida en que el sujeto la acepta como tal una vez ha aflorado: en esto, la confesión religiosa y las psicoterapias inductoras desarrolladas bajo el totalitarismo persiguen en escalas diferentes y con objetivos diversos que los sujetos reconozcan al final del proceso que, en el fondo, aceptan sinceramente ser así (ver Ruff 1958).

La sinceridad parece revelarse mediante la interfaz entre determinados formatos de enunciación interna y de inducción externa: dependiendo de estos, la verosimilitud se gradúa. Recordemos que todo régimen de verdad descansa sobre un sistema de crédito en un marco determinado. La lingüística y la filosofía modernas han puesto una especial atención en este punto. Para Jürgen Habermas, lo verosímil no es otra cosa que una articulación de sinceridades y autoridades, una «pretensión de validez» con una genealogía propia que hace posible su apariencia de realidad (Habermas 1994). Tzvetan Todorov o Julia Kristeva insistieron en que el surgimiento de lo verosímil en el pensamiento clásico no tenía una relación con lo real o lo verdadero, sino con lo que la mayoría de la gente cree que es lo real, dicho de otro modo, con la opinión pública autorizada, la doxa (Todorov y Kristeva 1972). Es por ello que cualquier sabotaje de los formatos de verdad pasará primero por el cuestionamiento de las formas de sinceridad en unos marcos de sentido concretos. En esto, Boris Groys o Eliseo Verón han sido precisos en su diagnóstico, al analizar la 


\section{artnodes}

http://artnodes.uoc.edu

La gestión matemática de la sinceridad...

fenomenología de la sospecha en la esfera de los modernos medios de comunicación en términos de «confianzas» y no de «certezas» (Groys 2008; Verón 2000), otorgando un papel esencial al estudio de los nuevos modos de crear un «decir veraz» y un «mirar suspicaz», en un espacio dominado por una ciencia que ha hecho suya la filosofía pragmatista del lenguaje propugnada por John Dewey, William James o Richard Rorty, cuya máxima era que lo verdadero es solo aquello que funciona (Engel 2008; Rorty 1991; James 1980) y cuya lógica interna (manifiestamente útil) es capaz de facilitar los objetivos de individuos y grupos sociales en pos de su bienestar.

¿Qué sucede con los mecanismos de equivalencia entre sinceridad y veracidad en la era de los social media y de los algoritmos que los rigen?

\section{Algoritmo y competencia social}

«Me llamo Morrón, mido treinta arsinas en cada dirección y, en efecto, desvalijo a los viajeros de sus cosas valiosas, pero de manera científica y moderna, es decir: me llevo secretos de gran valor, tesoros de ciencia, verdades auténticas y, en general, toda información valiosa. ¡Venga, pues! A soltar lo que os digo, porque si no, silbo. Cuento hasta cinco: uno, dos, tres...» (Lem 2005, 141).

Uno de los aspectos que pronto hay que tener presente es la transformación o desplazamiento de los espacios (o interfaces) dedicados a facilitar el encuentro entre lo interior y lo exterior, entre el formato de enunciación de un sujeto sincero y el mecanismo exterior que lo valida. «Extimidad» es un término creado por Jacques Lacan en 1969 para definir la existencia, dentro del ámbito más íntimo, de un «cuerpo extraño», aquello que es externo al individuo y con lo que se identifica. Según Lacan, «es lo más íntimo que estoy constreñido a no poder reconocer más que fuera» (Lacan 1990, 88). Más recientemente, el término ha ganado nuevos sentidos, en especial destinados a describir la exposición pública de la vida privada para producir feedback y retroalimentar un relato que configure verosímilmente al sujeto. La extimidad, así, es el deseo de revelar públicamente algunos aspectos de lo más íntimo a fin de fortalecer un discurso propio de sinceridad (Waelder 2011; Casilli 2011; Tisseron 2001; Gauchet 2000). El sujeto, privado de una vida social presencial que pasa a ser paulatinamente telemediada, gestionada a distancia y cuya proxémica es virtual (Hall 2003), se ve abocado a abandonar la profundidad y explorar la superficie, mediante una construcción vivencial de la emoción y de la experiencia social, en busca de una conducta y un hacer productivos, que mantengan el sentido y que refuercen su inserción en la doxa aceptada, incluso cuando esta se fundamenta en la exposición permanente de una contra-doxa o paradoja. Los diarios íntimos pasan a ser diarios éxtimos (Sibilia 2013). Ese es el trasfondo en el que, por ejemplo, la red social Sarahah cobró forma.
Sarahah es un servicio de red social que proporciona comentarios anónimos de una persona con el fin de «obtener una reacción sincera por parte de sus amigos y sus compañeros de trabajo» y así «ayudar a la gente a desarrollarse por sí misma de forma constructiva» (Binns 2017). El término árabe «Sarahah» significa «sinceridad». Fue creado a finales de 2016 y alcanzó éxito mundial a mediados de 2017, con más de 300 millones de usuarios. Sarahah está aparentemente diseñado para que las personas puedan componer su relato «interior» a partir del feedback social de unos testimonios que funcionan como árbitros de su sinceridad. En este sentido, los testimonios operan como fuente externa de veridicción, de forma más o menos análoga a la establecida en un juicio. El problema es que la aplicación ha generado graves tensiones, ya que los usuarios quedan expuestos a criticas públicas, que revelan las paradojas entre lo que se supone que ella o él deberían ser ante los demás y lo que los demás desean que sea, amén de los casos de acoso que el servicio fomenta.

Aquí, el mecanismo «externo» del dispositivo de veridicción adopta una condición presencial, aunque anónima (como en el caso de las delaciones fomentadas por los regímenes policiales escópicos), que tiene una función validatoria que depende tanto de la presencialidad del (d)enunciante, como del código de productividad impuesto sobre una comunidad. Sarahah, por consiguiente, no es en este aspecto un sistema del todo exógeno, razón por la cual su lógica todavía se percibe como aleatoria y regida por unas leyes colectivas vinculantes. $Y$ es en esta fina frontera donde el algoritmo se propone como epifanía de un nuevo régimen de credibilidad que pretende objetivar científicamente el proceso de autenticación de la sinceridad, desahuciando lo que de subjetivo tiene el proceso.

La precisión aparentemente objetiva e imparcial del algoritmo dice garantizar hoy mejor que nada el clásico contrato de veridicción. En computación, un algoritmo se define por ser un método mediante el cual se lleva a cabo una tarea a través de secuencias de pasos o instrucciones. Como tal, un algoritmo es una abstracción dotada de una existencia autónoma. Sin embargo, para que el algoritmo llegue a ser software social, «debe obtener su poder como artefacto social o cultural y proceso por medio de una adaptación cada vez mejor a los comportamientos y a los cuerpos que acontecen en su exterior» (Fuller 2008). Pero, ¿es el algoritmo el que se adapta a la exterioridad, o hemos llegado al proceso contrario, en el que los acontecimientos exteriores emulan el algoritmo? Para el filósofo Franco «Bifo» Berardi, se trata más bien de lo segundo: las palabras deben encajar en el proceso lógico de las máquinas, asimilando toda forma de sensibilidad y empatía en un juego de lógica productiva (Berardi 2017). Para Berardi, el lenguaje matemático es la forma más extrema de apropiación del lenguaje en la semiocracia (el gobierno del lenguaje y la economía): la producción de bienes materiales e inmateriales puede traducirse en una combinación y recombinación de información (algoritmos, figuras, diferencias digitales). Como el dinero, que es un equiva- 


\section{artnodes}

http://artnodes.uoc.edu

La gestión matemática de la sinceridad. .

Iente abstracto, no el significante de un referente fijo, las palabras devienen también huérfanas de sentido; el lenguaje que expresa el referente es suprimido, es desencarnado: solo son datos insertables en una cadena de plusvalía, que asumen una única función de rendimiento pragmático, de performance. Es la efectividad, y no el valor de la verdad, la nueva regla de ese lenguaje. El dispositivo principal de esta secuenciación es la «conexión». A diferencia de la «conjunción» que «requiere un criterio semántico de interpretación», un juego de sentidos, la «conexión» requiere únicamente un criterio sintáctico, un protocolo de traducción fijo y establecido a priori. Así, la transformación y la producción ya no acontecen en el campo de los cuerpos, sino en el de la pura interacción autorreferencial entre máquinas informáticas, que producen un «colapso del contexto». El algoritmo deviene, por consiguiente, un contrato privado de competencia pública, protegido por el derecho a la construcción subjetiva, que hace casi imposible el derecho a una objetividad común. Todo ello conlleva, en definitiva, una potente desorientación a la hora de elaborar racionalmente los fenómenos, favorecida por una nueva economía de la atención en cadena (armas de distracción masiva) (Citton 2014), que subraya con especial énfasis la deriva utilitarista del lenguaje, ya que fomenta en el receptor una selección de los fenómenos en función de la necesidad de construirse un relato verosímil que pueda sostenerse en el tiempo.

La matemática como lenguaje social se presenta, pues, como el marco veridictivo que permite objetivar cualquier forma de enunciado y presentarlo bajo unos meros parámetros cuantificables, y por lo tanto, veraces per se. Verdad y falsedad, en este nuevo régimen, ya no operan delimitados por lenguajes contextuales, sino como piezas de un puzle que encajarán gracias a la capacidad productiva del programa, que siempre será capaz de encontrar a sus parejas. Esta es una de las razones principales para el surgimiento estelar de las fake news en la actual esfera comunicativa automatizada. Cuando Italo Calvino apuntó, en un ensayo titulado «Cibernética y fantasmas» (1967), que «una cosa no se puede saber cuando las palabras y los conceptos para decirla y pensarla no han sido aún utilizados en esa posición, no han sido aún colocados en ese orden, en ese sentido", ya parecía intuir que la inteligencia artificial aplicada al lenguaje llegaría a ser capaz de prever y proveer toda posible forma de enunciado, acabando de una vez por todas con la posibilidad literaria de un lenguaje de los sentidos dispuesto a «asomarse al borde de lo decible», «fuera del vocabulario» (Calvino 2013, 65). En el código, no hay palabras sueltas, libres, fantasmales, porque el algoritmo es capaz de formular siempre la palabra que falta para acabar el rompecabezas que persigue: es arte y parte del dispositivo veridictivo: el objetivo es la producción y el mecanismo, una pura sintaxis lógica que ya no atiende a exterioridades. Esa objetividad radical es lo que hace de la posverdad un proyecto político con una competencia pública absolutista.

Los cuerpos reales y biológicos quedan relegados extramuros en este nuevo espacio de veridicción. A partir de trabajos como el de Jara Rocha y Femke Snelting en el proyecto Possible Bodies (2017-2018), ${ }^{4}$ acerca de los efectos de la semiótica médica (renders, prototipos y escáneres 3D) en el relato de los cuerpos, que los deja inútiles y ausentes, ya que solo su avatar es capaz de producir datos, de producir lenguaje con sentido, de contar «la verdad», podemos observar que el enunciado clásico del sujeto, necesario en el contrato tradicional de la veridicción, queda suspendido gracias a la aparente veracidad de la inteligencia artificial para «extraer» el verdadero interior del sujeto y poner sobre la mesa su grado de sinceridad, su competencia pública como usuario de la verdad productiva. Esa es la razón para que hoy el marketing hable de la sinceridad como un «valor añadido» en los social media. Veamos algunos ejemplos que describen bien esta condición.

Lenddo es una empresa en línea con base en Singapur, fundada en 2011, dedicada a realizar informes de «reputación» de clientes de países emergentes deseosos de adquirir un crédito bancario. Su método consiste en la aplicación de un complejo sistema de algoritmos de IA. La compañía declara como su principal objetivo el de «comprobar la transparencia, honestidad y compromiso» del solicitante. En 2017, había procesado más de 5 millones de solicitudes, siempre voluntarias. El proceso se inicia una vez el cliente ha contratado el servicio; seguidamente los algoritmos analizan tres patrones de fiabilidad:

1) Identidad («miramos quién es esa persona, la IP de su computadora, si escribe la verdad sobre su edad, su domicilio 0 su trabajo, o quiénes son sus conocidos. Porque será más fiable una persona cuyos conocidos también sean fiables») (Lenndo 2018)

2) Capacidad de pago («verificamos a través de su información cuál es su trabajo y cuáles son sus ingresos para calibrar si tendrá capacidad en algún momento de devolver el monto que pide. También si tiene un historial crediticio en el Buró de Crédito y si este es bueno o malo») (Lenndo 2018)

3) Voluntad de pago («comprobar que toda la información sea consistente y sin mentiras, que sea asiduo a las redes o qué comportamiento tiene en ellas con sus personas cercanas. Es decir, ver a qué personas mandas tus fotos, a quién le pones like... Tenemos probado que todos esos comportamientos acaban siendo la clave para saber el grado de compromiso de alguien») (Lenndo 2018)

4. Página web: https://possiblebodies.constantvzw.org/inventory/. 


\section{artnodes}

http://artnodes.uoc.edu

La gestión matemática de la sinceridad...

A la tradicional información bancaria (morosidad, historial de solvencia, bienes en propiedad, informes de vida laboral, clínica, etc.), Lenddo suma algoritmos que interpolan todos los datos derivados del uso del móvil, del ordenador, de las búsquedas en internet, de las redes sociales y de las compras digitales. Finalmente, estos sistemas establecen conclusiones psicométricas a partir del cotejo de los datos resultantes con el contenido del documento original de solicitud rellenado por el cliente. Todo el proceso tiene una duración de 3 minutos. El resultado se ofrece en formato numérico, con valores de 1 a 1000 puntos (Lenddo 2018; Abderrahim 2013).

A diferencia de los procesos de cruce de datos establecidos por la banca tradicional para estimar la solvencia, estos nuevos servicios digitales cuentan con la participación proactiva y voluntaria de los clientes, que ponen a disposición de la empresa mediadora toda la información personal y social derivada de su vida digital, a fin de proporcionar sus motivaciones y su compromiso de responsabilidad. Vemos, por consiguiente, que el contrato de veridicción clásico que establece la veracidad a partir del juicio a la sinceridad es ahora gestionado de una forma completamente nueva, ya que la sinceridad se determina mediante un conjunto instrumental predeterminado por su aparente infalibilidad, a través de un procedimiento meramente científico de «estimación» que viene a sustituir un ejercicio dialéctico.

Otro ejemplo similar es el proporcionado por Sesame Credit (también Ilamado Zhima Credit), ${ }^{5}$ un sistema de reputación crediticia fundado en 2015 por una empresa filial de Alibaba, el gigante chino de comercio electrónico. Los clientes reciben una puntuación basada en parámetros gestionados algorítmicamente sobre el historial bancario, los hábitos de consumo, las conexiones en redes sociales y datos de información personal. Los beneficios para los clientes que reciben puntuaciones altas consisten en «accesos más fáciles a préstamos, descuentos en cadenas hoteleras y empresas de alquiler de coches (que ofrecen reservas sin fianza), preferencia en las plataformas de búsqueda de trabajo, facilidad en la obtención de visados para salir del país», y, lo que la empresa más subraya, la exposición pública y notoria de los niveles de «confiabilidad» del usuario. Lo más relevante de este sistema es que tiene pleno apoyo del gobierno chino, cuya versión definitiva está ya siendo implantada bajo el nombre de Social Credit System, por la cual los ciudadanos chinos recibirán unas puntuaciones públicas basadas en su comportamiento político, social y financiero. El sistema está siendo diseñado para que las puntuaciones estén en relación con un baremo de «buenos y malos ciudadanos», llegando al extremo de penalizar a las personas que se relacionen con los usuarios con peor puntuación (Shazeda 2017). Un aspecto relevante y revelador de la aplicación de este sistema es que más de dos tercios de la población china lo ven con buenos ojos (Kostka 2018).
Germán Huici, al explorar la influencia de la teoría de juegos sobre las formas de interacción social, ha señalado que los comportamientos condicionados por los algoritmos tienden a reducir las opciones de elección a meros menús, con «opciones limitadas y variables controladas», como en los interfaces gráficos de usuario. Huici recuerda que los ensayos desarrollados por Morgenstern y Von Neumann precisaban que los modelos debían ser: 1) conformes a la realidad; 2) no ser complicados, para facilitar su manejo; 3) factibles de expresarse matemáticamente. Las encuestas, los exámenes de opción, o los tests psicológicos respondieron en su día a esas necesidades, que a su vez espejaron los modelos interficiales informáticos a partir de la década de 1990. Todo ello conduce a un «escenario artificial», a «una ilusión de coherencia» que se disfruta porque «mitiga la angustia ante la ausencia de orden» y porque se presenta como «la realidad redescubierta en su verdadera esencia» (Huici 2016, 69-74).

Ello implica, de nuevo, una nueva perspectiva sobre la verdad como predicción: «la realidad esconde un código que permite predecir su comportamiento» (Huici 2016, 70-71). La lógica interna y fantasmal del oráculo, de la «estimación» (pura exterioridad autorreferencial) parece reverdecer en la nueva lógica de la inteligencia artificial, por la cual la lógica del radar sustituye a la del objeto, ya que la pantalla es el único espacio capaz de aportar un relato con sentido. El contrato de veridicción queda aquí profundamente alterado y aparece un nuevo redactado que bien puede constituir un «nuevo régimen de verdad», basado en «la paralización de cualquier indicio de contradicción» (Sadin 2018, 81). La necesaria performance de la sinceridad (del acto de decir veraz) pasa a ser examinado de forma automatizada mediante un código ajeno, cuyo marco lingüístico solo responde a un conjunto de probabilidades y patrones, todos ellos programados para determinar grados de productividad de los sujetos en relación con lo que hacen los demás, a lo que es considerado «normal» 0 «estandarizado».

La importancia, en este punto, de la evolución de la estadística es notorio, puesto que el término «normal» surgió en el campo de los estudios probabilísticos (especialmente patológicos, véase la Antropología criminal) en el primer tercio del siglo xIX a fin de establecer estándares en lo tocante a los seres humanos. Fue el descubrimiento de la física del siglo xx de que el mundo no está sujeto al determinismo, sino que es producto constante del azar, lo que favoreció la progresión de las ciencias estadísticas encaminadas al establecimiento de probabilidades: el análisis de riesgos. La búsqueda de patrones se hizo perentoria, no sólo en las ciencias naturales y estadísticas, sino también en las humanas. Wilhelm Wundt, uno de los fundadores de la psicología cuantitativa escribió en 1862: «Es la estadística lo que primero demostró que el amor sigue leyes

5. Página web: https://www.xin.xin/\#/home. 


\section{artnodes}

http://artnodes.uoc.edu

La gestión matemática de la sinceridad...

psicológicas» (Hacking 2006, 19). Freud también hizo suyo el sistema de patrones (o síntomas) y, en general, el psicoanálisis ha operado mediante la recolección permanente y cruzada de los datos de los pacientes (Álvarez Huitrayao 2016). De hecho, la IA ya hace años que se está utilizando en las técnicas psicoanalíticas (Spruiell 1993).

lan Hacking ha demostrado que hubo que inventar nuevas técnicas de clasificación y de enumeración a cargo de nuevas burocracias de la autoridad, «para que la gente entrara convenientemente en ellas y pudiera ser contada y clasificada. La recolección sistemática de datos sobre las personas afectó no sólo la manera en que concebimos una sociedad, sino también las maneras en que describimos a nuestros semejantes», hasta el punto de que las leyes estadísticas acaban transformando los hechos mismos que los patrones analizan, produciéndose así el llamado «fatalismo estadístico», cuando «las alteraciones limitan la libertad de los grupos sociales para producir azar» (Hacking 2006, 19-20 y 179). Este mismo efecto ha sido sobradamente señalado en la aplicación de los modelos algorítmicos en el sistema de prisiones y en la predicción de crímenes en Estados Unidos (0’Neil 2018).

Pronto la estadística se acomodó al problema de la sinceridad. Condorcet (1785) o Laplace (1815) ya se habían ocupado de las posibilidades del uso del cálculo matemático para reducir las probabilidades de error en los jurados. Los problemas «empíricos» que presentaba un testigo en términos de probabilidad eran varios: ¿Puede prestarse crédito a esta persona?, ¿cómo se combina el testimonio de diferentes testigos con el mismo suceso?, ¿cómo se establece un jurado? (Hacking 2006, 132-156). El cálculo de probabilidades sirvió para ajustar la percepción sensible de los sujetos al marco programado de creencias, de modo que pudiera mantenerse su consistencia a toda costa, mediante un ejercicio de corrección. Es ya suficientemente conocido cómo Alphonse Bertillon, empleado en la prefectura policial de París, desarrolló en 1879 un sistema antropométrico de control de mediciones de detalles físicos, que pronto fue cruzado con sistemas de descripción para asegurar la «fiabilidad» de un aparato positivista de patrones e indicios a prueba de falsificaciones y engaños por parte de los criminales, y cuyo resultado final fueron los sistemas modernos de registro policial (Ginzburg 1980, 25-26). La cultura del algoritmo parece ofrecer una nueva versión de ese proceso. El «fatalismo estadístico", en este caso, se formula mediante la alteración de los marcos de enunciación, de manera que se limita la diversidad de formas de expresión para hacer posible que una Inteligencia Artificial pueda dirimir el grado de estandarización de nuestro «decir la verdad»; para que pueda establecer la dimensión del riesgo que representamos de no poder enunciar según los moldes de un lenguaje ajeno; para que premie el talento de quien lo aprende; para que pueda dirimir, en definitiva, el matiz corrector.

\section{¿Actualización o mutación?}

Nos preguntábamos más arriba si estamos, por lo tanto, frente a una potente actualización de los dispositivos de veridicción heredados del cientifismo positivista en forma de sistemas autorreferentes, 0 , por el contrario, acaso encaramos una mutación mayor. Probablemente, sea todavía pronto para dirimir esta cuestión. Indudablemente, la inteligencia artificial, aplicada a los sistemas de veridicción vinculados a las instituciones de justicia, parece actualizar de forma exponencial todo el conjunto de instrumentos desarrollados por la modernidad en relación con la colección, registro y proceso de información. Efectivamente, la potencia y velocidad de captación, cruce y aplicabilidad de los datos da a estos nuevos sistemas una utilidad gestora y presupuestaria difícilmente esquivable en la mentalidad productiva de las administraciones, públicas y privadas, que ven en ellos la confirmación de sus anhelos aparentemente garantistas, lo que, además, les proporciona una suerte de legitimidad pública, tanto en el sentido economicista como legal.

Sin embargo, el hecho de que los instrumentos antes vertebrados en el seno de los aparatos de justicia y seguridad hayan cruzado los tradicionales límites impuestos por la separación de poderes públicos, y hayan invadido escópicamente todos los ámbitos de la comunicación (comercio, crédito, ocio, trabajo...), y hayan disipando las fronteras entre lo privado y lo público, invita a pensar que este proceso puede alentar unas transformaciones que bien podrían considerarse paradigmáticas, abriendo así la puerta a la concepción de un sistema nuevo de veridicción.

De cualquier forma, las ventajas que presenta el machine learning en numerosos campos de investigación se convierten en riesgos potencialmente singulares con la introducción desregulada de la IA en el imaginario de la justicia, de la seguridad, de la gestión penal, y de la relación crediticia, ámbitos administrados mediante fuertes dispositivos de veridicción vinculados a las libertades públicas. Por un lado, en el caso crediticio, es un sistema que fomenta en el usuario el empleo de un lenguaje ad hoc para comunicar sus datos biográficos: la sinceridad se construye para adecuarse al dispositivo, con su lenguaje y sentidos propios. Por otro lado, como en el ámbito legal, al disponer ya de los datos antes de que el ciudadano los exponga (gracias al uso masivo de la biométrica y el big data), el sistema presenta la única opción posible que es la examinatoria, esto es, el juicio de las diferencias entre los datos y las explicaciones, convirtiendo al cuerpo social en permanentemente incriminable, ya que decimos sin cesar cosas falsas, convencidos de decir la verdad, o hacemos cosas sospechosas sin saber que lo son (en esto, las prácticas fake son siempre reveladoras). Es posible imaginar pronto una comunidad de «verdad enajenada». La comunidad, leída esta como los grupos humanos que vinculan su hacer a un sentido compartido del lenguaje, es descompetizada en sus formas de enunciación sincera, todas ellas muy complejas, y cuya lógica ahora 
se promete mensurar mediante procesos en los que el principio de conjetura da paso al de infalibilidad. No obstante, el fake de Joey Skaggs sobre las fantasías mecanicistas del sistema legal americano fue una forma hábil de advertir que no es tan fácil quitar todas las competencias, especialmente las de la risa y el ridículo cuando se ocupan del propio «contrato de veridicción», de los modos que nos dotamos para creernos los unos a los otros. La risa, al fin y al cabo, es uno de los formatos de veridicción más vinculantes que existen: un poderoso «decir veraz». Aunque, como todo, se puede simular, la risa es gravemente adherente y contagiosa.

Así, Stanislaw Lem publicó en 1965 la novela Ciberíada, dos de cuyos fragmentos hemos reproducido a lo largo de estas páginas. En ella, los dos protagonistas son Trurl y Clapaucio, una pareja de robots que viajan por el universo atendiendo diversos encargos. Uno de estos trabajos es la construcción de un «electrobardo», esto es, una gigantesca máquina lírica e inteligente. El resultado era que el monstruo «diezmaba a los poetas auténticos», no porque les dejara sin faena, sino porque les quitaba su competencia: todos los fenómenos del universo se habían convertido en poesía, gracias al poder del Electrobardo para abarcar cualquier posibilidad de sentido. Sólo le faltaba un detalle a la máquina: reír.

\section{Referencias bibliográficas}

Abderrahim, Jaled. 2013. «La sinceridad en las redes sociales como avalista de tu crédito». Yorokobu (20-12-2013). https://www. yorokobu.es/sinceridad-en-las-redes-sociales-como-avalistade-tu-credito/8/?offset=33

Aletras, Nikolaos; Tsarapatsanis, Dimitrios; Preotiuc-Pietro, Daniel y Lampos, Vasileios. 2016. «Predicting judicial decisions of the European Court of Human Rights: a Natural Language Processing perspective». PeerJ Computer Science 2:e93. https://doi. org/10.7717/peerj-cs.93

Álvarez Huitrayao, Manuel. 2016. La repetición, una interpretación psicoanalítica: Freud y Lacan. Tesis doctoral. Madrid: Universidad Complutense.

Berardi, Franco. 2017. Fenomenología del fin. Sensibilidad y mutación conectiva. Buenos Aires: Caja Negra.

Binns, Amy. 2017. «Sarahah, la 'aplicación de la sinceridad' que ha fomentado el ciberacoso». El País (28-8-2017). https://elpais.com/tecnologia/2017/08/23/actualidad/1503483935_042542.html

Calvino, Italo. 2013. «Cibernética y fantasmas». Punto y aparte. Madrid: Siruela.

Casilli, Antonio. 2011. Les liaisons numériques. Vers une nouvelle sociabilité?. París: Seuil.

Citton, Yves. 2014. Pour une écologie de l'attention. París: Éditions du Seuil.
Condorcet, Nicolas de. 1972. Essai sur l'application de l'analyse à la probabilité des décisions rendues à la pluralité des voix. New York: Chelsea.

Detienne, Marcel. 2004. Los maestros de verdad en la Grecia arcaica. Madrid: Sexto Piso.

Dewey, John. 2008. Logic: The Theory of Inquiry. Londres: Read Books. Eco, Umberto. 1996. Seis paseos por los bosques narrativos. Barcelona: Lumen.

Eco, Umberto. 1998. Serendipities: Language and Lunacy. Nueva York: Columbia University Press. https://doi.org/10.7312/ecou11134

Engel, Pascal. 2008. ¿Qué es la verdad? Reflexiones sobre algunos truismos. Buenos Aires-Madrid: Amorrortu.

European Commission for the Efficiency of Justice (CEPEJ). 2018. «European ethical Charter on the use of Artificial Intelligence in judicial systems and their environment». Consejo de Europa, Estrasburgo. https://rm.coe.int/ethical-charter-en-for-publication4-december-2018/16808f699c

Foucault, Michel. 1980. Microfísica del Poder. Madrid: La Piqueta.

Foucault, Michel. 2001. Fearless Speech. Los Angeles: Semiotext. Este volumen recoge las seis conferencias pronunciadas por el autor en la Universidad de California en Berkeley en el otoño de 1983, bajo el título «Discurso y verdad».

Foucault, Michel. 2004. El orden del discurso. Barcelona: Tusquets.

Foucault, Michel. 2014. Obrar mal, decir la verdad. Buenos Aires: Siglo XXI.

Fuller, Matthew (ed). 2008. Software Studies: A Lexicon. Cambridge: The MIT Press. https://doi.org/10.7551/mitpress/9780262062749.001.0001

Gauchet, Marcel. 2000. «A New Age of Personality: An Essay on the Psychology of our times». Thesis Eleven, $n^{0}$ 60: 23-41. https:// doi.org/10.1177/0725513600060000004

Ginzburg, Carlo. 1980. «Morelli, Freud and Sherlock Holmes: Clues and Scientific Method». History Workshop, n 9: 5-36. https://doi. org/10.1093/hwj/9.1.5

Greimas, Algirdas J.; Courtés, Joseph. 1982. Semiótica. Diccionario razonado de la teoría del lenguaje. Madrid: Gredos.

Groys, Boris. 2008. Bajo sospecha. Una fenomenología de los medios. Valencia: Pre-Textos.

Habermas, Jürgen. 1994. Historia y crítica de la opinión pública. Ciudad de México: Gustavo Gili.

Hacking, lan. 2006. La domesticación del azar. La erosión del determinismo y el nacimiento de las ciencias del caos. Barcelona: Gedisa.

Hall, Edward T. 2003. La dimensión oculta. Ciudad de México y Buenos Aires: Siglo XXI.

Huici, Germán. 2016. El Dios ausente. Iconografía y metafísica del capitalismo. Barcelona: Elba.

James, Williams. 1980. El significado de la verdad. Buenos Aires: Aguilar.

Kostka, Genia. 2018. «China's Social Credit Systems and Public 
Opinion: Explaining High Levels of Approval». SSRN. https://doi. org/10.2139/ssrn.3215138

Kristeva, Julia. 1972. «La productividad Ilamada texto». En Barthes et al. Lo verosímil. Buenos Aires: Tiempo Contemporáneo, 63-94.

Lacan, Jacques. 1990. El seminario. Libro 7: La ética del psicoanálisis (1959-1960). Barcelona: Paidós.

Laplace, Pierre-Simon. 2012. A Philosophical Essay on Probabilities. New York: Dover.

Lem, Stanislaw. 2005. Ciberíada. Madrid: Alianza.

Lendo. 2019. https://www.lenddo.com/index.html

Marzo, Jorge Luis. 2015. «La genealogía líquida de la interfaz». Artnodes, $n^{0}$ 16: 5-16. https://doi.org/10.7238/a.v0i16.2784

Marzo, Jorge Luis. 2018. La competencia de lo falso. Una historia del fake. Madrid: Cátedra.

Ministerio de Energía, Turismo y Agenda Digital. 2017. Nota de prensa «El Ministerio de Agenda Digital y el CGPJ firman un convenio para impulsar las tecnologías del lenguaje». Web del Ministerio: https:// www.mincotur.gob.es/es-es/gabineteprensa/notasprensa/2017/ documents/171013\%20np\%20convenio\%20minetad\%20cgpj.pdf

Molina, Sara. 2018. «La Inteligencia Artificial se asoma a la fase de consolidación en el sector legal». Consejo General de la Abogacía Española. https://www.abogacia.es/2018/12/10/la-inteligenciaartificial-se-asoma-a-la-fase-de-consolidacion-en-el-sectorlegal

Niler, Eric. 2019. «Can Al Be a Fair Judge in Court? Estonia Thinks So». Wired. https://www.wired.com/story/can-ai-be-fair-judgecourt-estonia-thinks-so/

0’Neil, Cathy. 2018. Armas de destrucción matemática. Madrid: Capitán Swing.

Pierce, Charles S. 1877. «The Fixation of Belief». Popular Science Monthly 12: 1-15. http://www.peirce.org/writings/p107.html

Revel, Judith. 2018. «From Truth as a Content to Truth as a Practice: Political Ethics of Parrhesia». Keynote en el II Congreso Internacional
Interface Politics. Organizado por GREDITS/BAU, Barcelona. Leído el 28 de noviembre de 2018. Actas en proceso de publicación. URL del congreso: http://www.gredits.org/interfacepolitics/es/ Rorty, Richard. 1991. Contingencia, ironía y solidaridad. Barcelona: Paidós.

Ruff, Lajos. 1958. La máquina de lavar cerebros. Madrid: Testigos de Hoy.

Tashea, Jason. 2017. «Courts are using Al to sentence criminals. That must stop now». Wired. https://www.wired.com/2017/04/ courts-using-ai-sentence-criminals-must-stop-now/

Sadin, Éric. 2018. L'Intelligence artificielle ou l'enjeu du siècle. Anatomie d'un antihumanisme radical. París: L'échappée.

Shazeda, Ahmed. 2017. «Cashless Society, Cached Data Security Considerations for a Chinese Social Credit System». The Citizen Lab: URL: https://citizenlab.ca/2017/01/cashless-society-cacheddata-security-considerations-chinese-social-credit-system/

Sibilia, Paula. 2013. La intimidad como espectáculo. Buenos Aires: Fondo de Cultura Económica.

Skaggs, Joey. 2006. «Solomon Project». Web del artista. https:// joeyskaggs.com/wp-content/uploads/2015/07/skaggs-solomonpressreleaseexpose-1-96-800.jpg

Spruiell, V. 1993. «Deterministic Chaos and the Sciences of Complexity: Psychoanalysis in the Midst of a General Scientific Revolution». Journal of the American Psychoanalytic Association, 41(1): 3-44. https://doi.org/10.1177/000306519304100101

Tisseron, Serge. 2001. L'intimité surexposée. París: Ramasay.

Todorov, Tzvetan. 1972. «Introducción». En R. Barthes et al. Lo verosímil. Buenos Aires: Tiempo Contemporáneo, 11-16.

Verón, Elise0. 2000. «Análisis inédito sobre un célebre caso de arte desmaterializado». Ramona, n 9-10: 46-50.

Waelder, Pau. 2011. «Lo íntimo es otro». En Pau Waelder(ed). Extimitat: Art, intimitat i tecnologia. Palma de Mallorca: Es Baluard, pp. 108-117. 


\section{artnodes}

\section{CV}

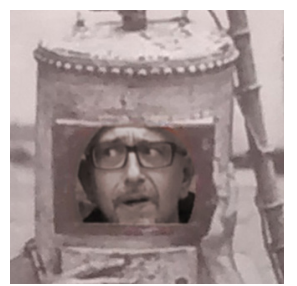

\section{Jorge Luis Marzo Pérez}

diga@soymenos.net

Doctor en Estudios Culturales, Traducción y Género

BAU, Centro Universitario

De Diseño de Barcelona

c/ Pujades, 11808005

Barcelona (España)

Profesor agregado a BAU Centre Universitari de Disseny de Barcelona Miembro de GREDITS (Grup de Recerca en Disseny i Transformació Social)

https://orcid.org/0000-0003-1238-8703 